\title{
Heart rate variability recovery after a skyrunning marathon and correlates of performance
}

\author{
Michaela Mertová ${ }^{1}$, Michal Botek ${ }^{1}$, Jakub Krejčíi,*, and Andrew J. McKune ${ }^{2,3}$ \\ ${ }^{1}$ Faculty of Physical Culture, Palacký University Olomouc, Olomouc, Czech Republic; ${ }^{2}$ Research Institute for Sport and \\ Exercise, University of Canberra, ACT, Australia; and ${ }^{3}$ School of Health Sciences, University of KwaZulu-Natal, Durban, \\ South Africa
}

Copyright: (C) 2017 M. Mertová et al. This is an open access article licensed under the Creative Commons Attribution License (http://creativecommons.org/licenses/by/4.0/).

Background: It is well known that vigorous physical activity induces functional changes in cardiac autonomic nervous system (ANS) activity that is sustained several hours after exercise. However, data related to ANS recovery after more extreme endurance events, such as skyrunning marathons, are still lacking. Objective: The aims of this prospective cohort study were firstly, to determine the ANS response to a SkyMarathon, and secondly, to examine correlates of run performance. Methods: Ten male skyrunners aged $37.2 \pm 9.2$ years were recruited. The race was performed at a mean intensity $85.4 \pm 3.7 \%$ of heart rate reserve, and lasted for $338 \pm 38 \mathrm{~min}$. Morning supine heart rate variability was measured at 10, 2 and 1 days before race, on the race day, at $5 \mathrm{~min}$ intervals for $30 \mathrm{~min}$ immediately post-race and then at $5 \mathrm{~h}$ and $30 \mathrm{~h}$ post. High-frequency power (HF, 0.15-0.50 Hz), low-frequency power (LF, 0.05-0.15 Hz), and square root of the mean of the squares of the successive differences (RMSSD) were calculated and transformed by natural logarithm (Ln). Results: Sympathovagal balance (Ln LF/HF) was most likely increased above baseline during the $30 \mathrm{~min}$ post-race and returned to baseline by $5 \mathrm{~h}$. Vagal activity (Ln RMSSD and Ln HF) was most likely decreased below baseline during the $30 \mathrm{~min}$ post-race and $5 \mathrm{~h}$ of post-race, and recovered to baseline by $30 \mathrm{~h}$. Race time correlated with resting heart rate $(r=.81)$, body mass index $(r=.73)$, maximal power output $(r=-.70)$, and maximal oxygen uptake $(r=-.61)$. Conclusions: The SkyMarathon elicited disturbances in ANS activity, with relative sympathetic activity increased up to $5 \mathrm{~h}$ post-race and vagal activity recovering by $30 \mathrm{~h}$. Resting heart rate, body mass index, maximal power output, and maximal oxygen uptake were associated with SkyMarathon performance prediction.

Keywords: vagal activity, vigorous exercise, fatigue, endurance, cardiac stress, environmental conditions

\section{Introduction}

Skyrunning is a young, extreme sport variation of mountain running that takes place at high altitudes above $2000 \mathrm{~m}$. For countries whose altitude is below the specified altitude, an exception to the rules may be applied to national championships and a minimum of $2000 \mathrm{~m}$ vertical climb is required (International Skyrunning Federation, 2017). Skyrunning includes steep inclines and climbing across difficult terrain, where race times range from 3 to $20 \mathrm{~h}$ (Roi, Monticone, Salvoni, Sassi, \& Alberti, 2015). Route profiles include uphill (concentrically biased) and downhill

\footnotetext{
* Address for correspondence: Jakub Krejčí, Department of Natural Sciences in Kinanthropology, Faculty of Physical Culture, Palacký University Olomouc, třída Míru 117, 77111 Olomouc, Czech Republic. E-mail: jakub.krejci@upol.cz
}

(eccentrically biased) sections that place demands on musculoskeletal, cardiorespiratory and metabolic systems (Baron et al., 2009; Fridén \& Lieber, 2001; Vernillo et al., 2014).

Acute physiological adjustments to exercise are mediated throughout functional changes in the autonomic nervous system activity and catecholamine production (Åstrand, Rodahl, Dahl, \& Strømme, 2003). The spectral analysis (SA) of heart rate variability (HRV) is commonly considered to be a non-invasive method for the quantification of cardiac autonomic nervous system (ANS) related to the sinoatrial node (Akselrod et al., 1981). The high-frequency power (HF) is entirely modulated by cardiac vagal activity (Pomeranz et al., 1985; Warren, Jaffe, Wraa, \& Stebbins, 1997). The low-frequency power (LF) is associated with baroreflex activity and the bilateral effect of sympathetic and vagal activity on the sinus node 
(Task Force of the European Society of Cardiology and the North American Society of Pacing and Electrophysiology, 1996), and LF/HF ratio reflects the sympathovagal balance (Ernst, 2014). Besides spectral variables, a time domain variable, specifically the square root of the mean of the squares of the successive differences (RMSSD), considered as an index of vagal activity (Buchheit, 2014) has become very popular among researchers (Flatt, Esco, \& Nakamura, 2017; Nakamura et al., 2015; Plews, Laursen, Stanley, Kilding, \& Buchheit, 2013) for field HRV analysis. One of the advantages of RMSSD is that it is less sensitive to low breathing frequency than spectral high-frequency power (Penttilä et al., 2001).

A progressive increase in exercise intensity causes a significant decrease in vagal activity (Arai et al., 1989; Botek, Stejskal, Krejci, Jakubec, \& Gaba, 2010; Perini, Orizio, Baselli, Cerutti, \& Veicsteinas, 1990; Stejskal et al., 2001), and the sympathoadrenal system starts to dominate at an intensity around $40-50 \% \mathrm{VO}_{2} \max$ (Hautala et al., 2003). After exercise, cardiac vagal activity is initially reduced within minutes to hours after exercise (Botek, Stejskal, \& Neuls, 2008; Buchheit, Papelier, Laursen, \& Ahmaidi, 2007; Mourot et al., 2015; Stanley, Peake, \& Buchheit, 2013). Previous research has proposed that the time course of vagal recovery depends primarily on exercise intensity (Arai et al., 1989; Stejskal et al., 2001), and/or cardiorespiratory performance (Hautala et al., 2001; Seiler, Haugen, \& Kuffel, 2007). However, exercise duration should also be considered as a factor that influences ANS recovery (Kaikkonen, Hynynen, Mann, Rusko, \& Nummela, 2010). A number of studies have focused on ANS recovery in response to distance running e.g. half-marathon (Botek, Stejskal, Šafář, \& Smékal, 2011; Dalla Vecchia, Traversi, Porta, Lucini, \& Pagani, 2014), marathon (Botek et al., 2008; DaniłowiczSzymanowicz et al., 2005), a $46 \mathrm{~km}$ wilderness trail run (Bernardi, Passino, Robergs, \& Appenzeller, 1997), and/or a cross-country skiing run (Hautala et al., 2001; Mourot et al., 2015). However, data relating to recovery HRV after skyrunning is limited.

The primary aim of this study was to assess acute and long-term (up to $30 \mathrm{~h}$ ) HRV recovery after a SkyMarathon race. Determining the time for recovery and severity of the HRV disturbance post-race may provide practical information to guide training preparation, periodization and recovery protocols in this young, developing sport. Skyrunning performance may be associated with similar physiological variables that correlate with road marathon running performance such as running economy, $\mathrm{VO}_{2} \max$ and anaerobic thresholds level (Noakes, 2000). However, skyrunning is performed in harsher environmental and terrain conditions compared with marathon running which increases the possibility of different associations between variables. Therefore, the secondary aim of the study was to determine relationships between a number of physiological and somatic characteristics and skyrunning performance.

\section{Methods}

\section{Participants}

Thirteen physically fit, healthy, amateur, male skyrunners volunteered to participate in the study. Due to injuries during the race, only ten runners crossed the finish line, and were therefore included in the final analyses. All runners were nonsmokers, and were not taking any medication. All subjects had previous mountain running and long-distance racing experience. Total kilometers run in the month leading up to the SkyMarathon were $270 \pm 80 \mathrm{~km}$. Training preparation was carried out individually without supervision. The somatic and physiological characteristics of the 10 athletes are presented in Table 1. The research design was approved by the Ethics Committee at the Faculty of Physical Culture, Palacký University Olomouc and conformed to the recommendations from the Declaration of Helsinki. All subjects provided written informed consent.

\section{Experimental procedures}

The experiment took place during April and early May 2015. An overview of the study design is shown in Figure 1. The race was held on the $2^{\text {nd }}$ May, 2015. All subjects underwent a pre-race laboratory screening fifteen days before the competition to determine the presence of any health issues that may prevent them from performing maximal exercise testing. Alcohol consumption was strictly forbidden during the study, and subjects were requested to avoid vigorous training for two days before the pre-race laboratory screening.

HRV measurement was performed at home 10, 2 and 1 days before the race. On race day, HRV measurement was carried out under field testing conditions in quiet chalet room reserved for this purpose. Following the assessment, the SkyMarathon started. Within 5 min of the race completion, HRV was measured in the supine position for $30 \mathrm{~min}$. In addition, HRV was further assessed in a quiet chalet room on average $5 \mathrm{~h}$ post-race and at home on average $30 \mathrm{~h}$ post-race.

\section{Pre-race laboratory testing}

The subjects had their height, weight, and body mass index (BMI) measured using the SOEHNLE 7307 (Leifheit, Nassau, Germany). Body fat percentage was determined using bioimpedance analysis (InBody 720, InBody, Seoul, South Korea). 
Table 1

Somatic and physiological characteristics of the tested group $(N=10)$ and correlations with race time

\begin{tabular}{lccc}
\hline Variable & Mean $\pm S D$ & $r$ & MBI \\
\hline Age $($ years $)$ & $37.2 \pm 9.2$ & .33 & $30 / 69 / 1$, possibly trivial \\
Height $(\mathrm{cm})$ & $178.8 \pm 3.6$ & .09 & $11 / 84 / 5$, likely trivial \\
Weight $(\mathrm{kg})$ & $73.1 \pm 7.0$ & .55 & $58 / 42 / 0$, possibly positive \\
BMI $\left(\mathrm{kg} \cdot \mathrm{m}^{-2}\right)$ & $22.8 \pm 1.5$ & .73 & $84 / 16 / 0$, likely positive \\
Body fat $(\%)$ & $11.2 \pm 3.9$ & .49 & $49 / 51 / 0$, possibly trivial \\
$\mathrm{VO}_{2} \max \left(\mathrm{ml} \cdot \mathrm{kg}^{-1} \cdot \mathrm{min}^{-1}\right)$ & $61.2 \pm 4.3$ & -.61 & $0 / 34 / 66$, possibly negative \\
Pmax $\left(\mathrm{W} \cdot \mathrm{kg}^{-1}\right)$ & $6.21 \pm 0.64$ & -.70 & $0 / 20 / 80$, likely negative \\
ANT $\left(\% \mathrm{VO}_{2} \max ^{2}\right.$ & $89.5 \pm 5.4$ & -.34 & $1 / 69 / 30$, possibly trivial \\
HRmax $\left(\right.$ beats $\left.\cdot \min ^{-1}\right)$ & $184 \pm 12$ & .01 & $8 / 85 / 7$, unclear \\
\hline
\end{tabular}

Note. $\quad r=$ Pearson's correlation coefficient; $\mathrm{MBI}=$ magnitude based inference for $r$; $\mathrm{BMI}=$ body mass index; $\mathrm{VO}_{2} \max =$ maximal oxygen uptake; $\mathrm{Pmax}=$ maximal power output; $\mathrm{ANT}=$ anaerobic threshold $; \mathrm{HRmax}=$ maximal heart rate.

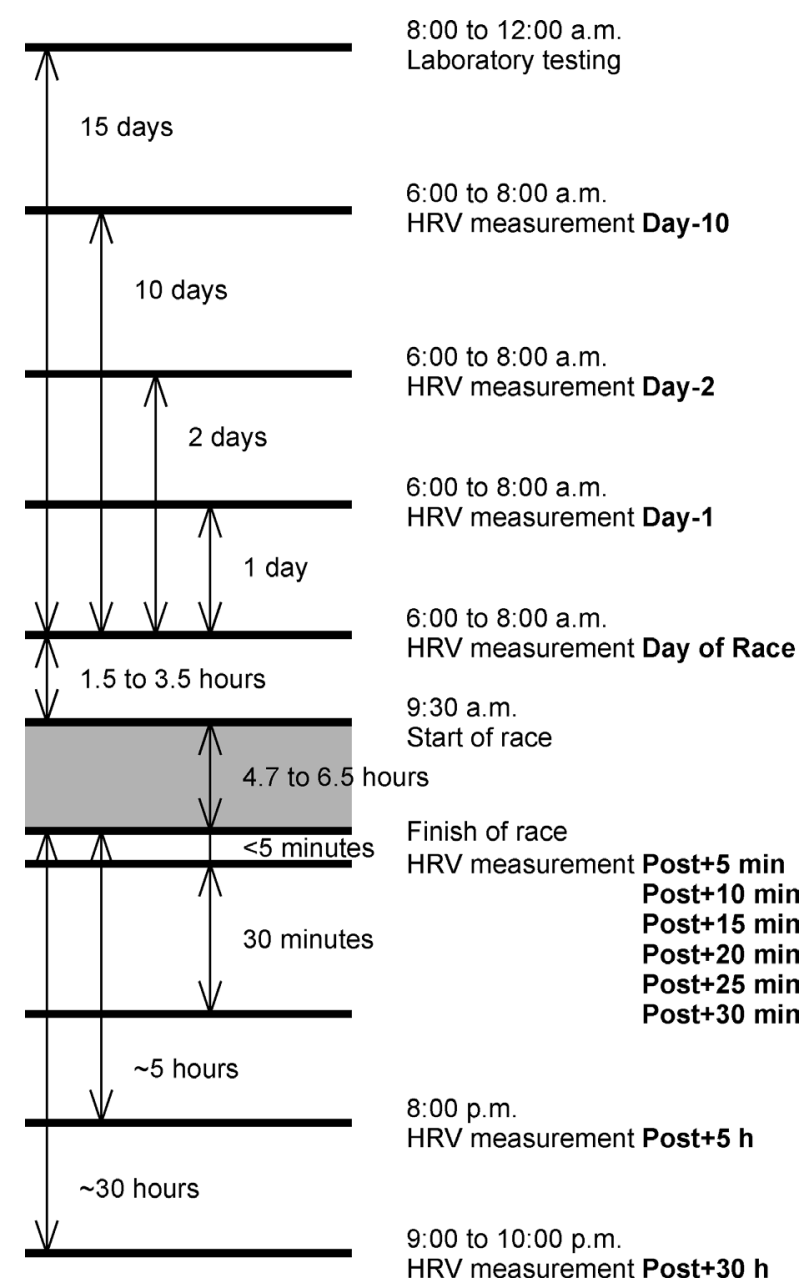

Figure 1. Overview of the study design and depiction of HRV measurements. The time axis is not proportional with time values to capture large differences in time values.
Each subject underwent an incremental running test on a treadmill (Lode Valiant, Groningen, Netherlands) in order to obtain maximal oxygen uptake $\left(\mathrm{VO}_{2} \max \right)$ and maximal heart rate (HRmax). The exercise protocol consisted of a 4-min warm-up period (3-min at $8.0 \mathrm{~km} \cdot \mathrm{h}^{-1}$ with $0 \%$ inclination and a further 1 -min at the same speed with a $5.0 \%$ inclination). The speed was then increased to $10.0 \mathrm{~km} \cdot \mathrm{h}^{-1}$ for $1 \mathrm{~min}$ with the gradient kept at $5.0 \%$. Then, for each minute thereafter speed increased by $1.0 \mathrm{~km} \cdot \mathrm{h}^{-1}$ with the gradient at $5.0 \%$ up to maximal speed of $16.0 \mathrm{~km} \cdot \mathrm{h}^{-1}$. From this stage only inclination increased by $2.5 \%$ every min until exhaustion. Breath-by-breath ventilation and gas exchange were continuously analyzed (Geratherm, nSpire Health, Oberthulba, Germany) during the exercise with the data averaged to 30 seconds for analysis. Gas and flow analyzers were recalibrated before and after each testing using gases of known concentration and a $3 \mathrm{~L}$ calibration syringe. During the test, the ambient temperature was maintained between 22 to $24{ }^{\circ} \mathrm{C}$ with relative humidity between $40-60 \%$.

The following criteria were used to document that $\mathrm{VO}_{2}$ max was achieved: i) a lack of increase in $\mathrm{VO}_{2}$ upon an increase in work rate, ii) respiratory exchange ratio >1.10 (Shephard \& Åstrand, 1992). $\mathrm{VO}_{2} \max$ was recorded as the highest $\mathrm{VO}_{2}$ value in the final 30 seconds of the test (Millet et al., 2003). Heart rate (HR) responses were monitored continuously during the incremental running test using a WearLink heart rate sensor (Polar, Kempele, Finland). Maximal power output (Pmax) was calculated according to following formula (American College of Sports Medicine, 1986): $P=(0.2 s+0.9 s g+3.5) / 10.5$, where: $P$ is relative power output $\left(\mathrm{W} \cdot \mathrm{kg}^{-1}\right), s$ is speed $\left(\mathrm{m} \cdot \mathrm{min}^{-1}\right)$, and $g$ is fractional grade. 


\section{HRV measurement}

HRV measurement was performed in the morning between 6 and 8 a.m., except for the measurements taken after the race. With the runners lying supine, RR intervals were recorded over $12 \mathrm{~min}$ in a quiet room using a Polar RS800CX heart rate monitor (Polar, Kempele, Finland). Within 5 min of the race completion, supine RR intervals (Polar RS800CX) were recorded for $30 \mathrm{~min}$ in a quiet room, where the ambient temperature ranged from 20 to $24{ }^{\circ} \mathrm{C}$. All RR intervals were subsequently imported into Polar Pro Trainer 5 software, and then imported in text format into diagnostic system DiANS PF8 (DIMEA Group, Olomouc, Czech Republic) for further HRV analysis. After the RR intervals had been imported, all premature ventricular contractions, missing beats, and any artefacts were manually filtered. For further HRV analysis, only 300 artefact-free RR intervals were selected. A SA HRV was used to assess ANS activity and was performed using the Fast Fourier Transformation. The following spectral variables were computed: high-frequency power (HF, 0.15-0.50 Hz), low-frequency power (LF, 0.05-0.15 $\mathrm{Hz}), \mathrm{LF} / \mathrm{HF}$ ratio, and total power $(\mathrm{PT}=\mathrm{LF}+\mathrm{HF})$. Mean heart rate in the supine position (HRsup) and RMSSD were also calculated. For correlation analysis, the HRsup on the day of the race was considered to be the resting heart rate (HRrest).

\section{Race measures}

The SkyMarathon Perun 2015 race was performed in the Beskydy Mountains at an altitude ranging from $424 \mathrm{~m}$ to $1027 \mathrm{~m}$ above sea level (Figure 2), as a part of the Czech National Championship 2015. The race started at 9:30 a.m. with the ambient temperature $10{ }^{\circ} \mathrm{C}$, humidity $87 \%$, and air pressure $1,014.2 \mathrm{hPa}$. Runners covered a distance of $\sim 42 \mathrm{~km}$, with an ascent distance $3.15 \mathrm{~km}$ and a descent distance $2.85 \mathrm{~km}$. During the race, subject HR responses were continuously

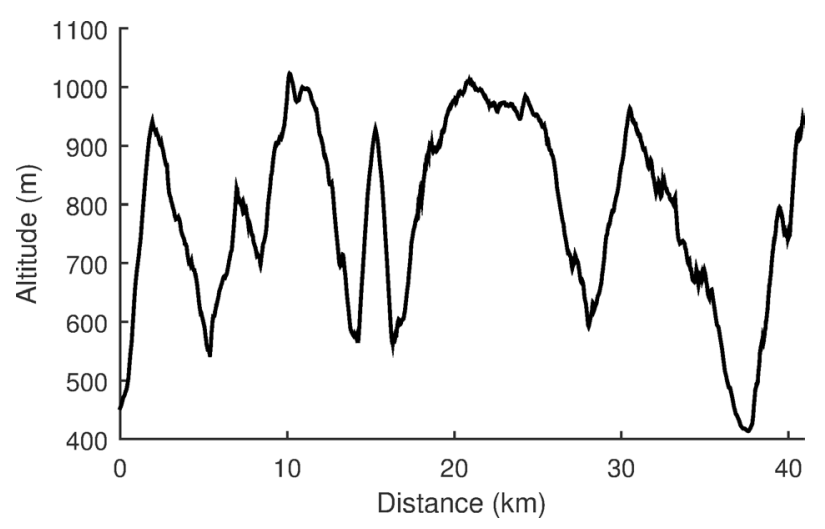

Figure 2. Terrain profile of race 2015. sampled using the Polar RS800CX heart rate monitor with recording rate at 5 seconds.

\section{Statistical analysis}

Data are presented as arithmetic means \pm standard deviations unless otherwise stated. Normality and homoscedasticity of data were checked using the Kolmogorov-Smirnov test and Levene's test, respectively. Because all HRV variables were not normally distributed, all statistical analyses were performed on natural logarithm ( $\mathrm{Ln}$ ) transformed values. The hypothesis of homoscedasticity was rejected for all Ln transformed HRV variables therefore using an ANOVA was not appropriate. This problem was overcome using a set of 11 separate $t$-tests. The reference value was the morning measurement from the day of the race (the measurement marked "Day of Race" in Figure 1). The remaining 11 measurements were expressed as differences calculated as the selected value minus the reference value. The $p$-value of each difference was calculated using one-sample $t$-test. Linear relationships between variables were evaluated using Pearson's correlation coefficient $(r)$. The magnitude of $r$ was interpreted according following thresholds adopted from Hopkins, Marshall, Batterham, and Hanin (2009): $<.5$ (trivial), $\geq .5$ (large), $\geq .7$ (very large), and $\geq .9$ (extremely large). We did not use a finer scale for magnitudes $<.5$ because we considered magnitudes $<.5$ to be impractical for prediction purposes.

Because $p$-values are sample-size dependent and do not inform on magnitude of effects, we used magnitude-based inference proposed by Batterham and Hopkins (2006). Magnitude-based inferences were based on calculating $90 \%$ confidence interval (CI) that defines a range representing the uncertainty in the true value. A three-level scale: substantially positive, trivial, and substantially negative, was defined by the smallest worthwhile change (SWC). Chances that the true values were substantially positive, trivial, and substantially negative, were calculated by comparing the CI to the three-level scale. If the chance of substantially positive or substantially negative was simultaneously $>5 \%$, the true value was deemed as unclear. Otherwise the chances were labeled quantitatively as follows: $25-75 \%$, possibly; 75-95\%, likely; 95-99.5\%, very likely; and >99.5\%, most likely (Hopkins et al., 2009). For each participant, intra-individual standard deviation was calculated from three consecutive days before the race (measurements marked "Day-2", "Day1", and "Day of Race" in Figure 1). We set the SWC for difference to the pooled standard deviation calculated from all intra-individual standard deviations (Buchheit, 2014). We set the SWC for $r$ to 0.5 . 
Statistical analyses were performed using MATLAB 8.4 with Statistics Toolbox 9.1 (MathWorks, Natick, MA, USA). A spreadsheet (Hopkins, 2007) was used to convert a $p$-value into CI and chances.

\section{Results}

The mean HR during the race was $157 \pm 7$ beats $\cdot \mathrm{min}^{-1}$, which is equal to $85.4 \pm 3.7 \%$ of heart rate reserve. The race time (RT) of the tested group was $338 \pm 38 \mathrm{~min}$ and demonstrated a high inter-individual variability with the best runner completing in $280 \mathrm{~min}$ and the slowest runner taking $112 \mathrm{~min}$ longer. Correlations between achieved RT and somatic, physiological, and HRV variables are presented in Table 1 and Table 2. The largest four magnitudes of correlations were found in the following variables (Figure 3$)$ : HRrest ( $r=.81$, likely positive), BMI $(r=.73$, likely positive), Pmax $(r=-.70$, likely negative), and $\mathrm{VO}_{2} \max (r=-.63$, possibly negative).

Values of HRsup and HRV variables on the day of the race are presented in Table 2. Values of SWC used for analysis of HRsup and HRV changes are presented in Table 2. As shown in Figure 4, HRsup was most likely increased within the acute recovery period (up to 30 min post-race) compared to the pre-race level. All studied HRV variables except $\mathrm{Ln} L \mathrm{~L} / \mathrm{HF}$ were very likely or most likely decreased within the acute recovery period. Ln LF/HF was very likely or most likely increased within the acute recovery period.

HRsup was most likely increased at $5 \mathrm{~h}$ post-race compared to the pre-race level. Ln RMSSD, Ln LF, Ln HF, and Ln TP were most likely, likely, most likely, and very likely, respectively, decreased at $5 \mathrm{~h}$ post-race. Only $\mathrm{Ln} \mathrm{LF} / \mathrm{HF}$ returned to pre-race levels at $5 \mathrm{~h}$ post-race, with the difference possibly trivial. HRsup and all studied HRV variables returned to pre-race levels at $30 \mathrm{~h}$ post-race, with differences possibly or very likely trivial.

\section{Discussion}

This research was primarily designed to assess the cardiac ANS response to a SkyMarathon race during $30 \mathrm{~h}$ of recovery, and secondly to identify somatic and physiological characteristics associated with skyrunning performance. The main findings of this research were that sympathovagal balance was altered in the first $30 \mathrm{~min}$ post-race but had returned to baseline by $5 \mathrm{~h}$. While cardiac vagal activity, estimated from Ln HF and Ln RMSSD, was significantly reduced in the $30 \mathrm{~min}$ and $5 \mathrm{~h}$ post-race and only recovered to baseline by $30 \mathrm{~h}$ post-race. In addition, non-trivial associations between achieved RT and HRrest, BMI, Pmax, and $\mathrm{VO}_{2} \max$ were found.

In the present study, the reduced Ln RMSSD and Ln HF of RR intervals, accepted indexes of cardiac vagal activity (Buchheit, 2014; Task Force of the European Society of Cardiology and the North American Society of Pacing and Electrophysiology, 1996), reflected an acute withdrawal of cardiac vagal modulation within the first 30 min post-race, while the increased $\mathrm{Ln} \mathrm{LF} / \mathrm{HF}$ ratio indicated a relative sympathetic dominance. A persisted sympathetic stimulation together with blunted vagal activity induced by the race (5.6 h with intensity $\sim 85 \%$ of heart rate reserve) was mirrored by the significantly increased HR compared to baseline within the first $30 \mathrm{~min}$ post-race. A drop in $\mathrm{Ln} \mathrm{LF} / \mathrm{HF}$ back to baseline at $5 \mathrm{~h}$ post-race showed an adjustment in the sympathovagal balance, however vagal activity remained blunted and only returned to baseline at $30 \mathrm{~h}$ post-race. Based on our results, we suggest that the return to pre-race levels for both cardiac vagal activity and resting HR after SkyMarathon is delayed compared with the sympathetic activity recovery. Whether skyrunners are more prone to cardiovascular health complications in the acute period postrace was not the focus of the present study. However,

Table 2

Means, standard deviations, and correlations with race time calculated from morning HRV measurement on the day of the race and smallest worthwhile changes (SWC) calculated from three consecutive days before the race

\begin{tabular}{lcccc}
\hline Variable & Mean $\pm S D$ & $r$ & MBI & SWC \\
\hline HRsup (beats $\left.\cdot \mathrm{min}^{-1}\right)$ & $51.1 \pm 6.7$ & .81 & $94 / 6 / 0$, likely positive & 3.0 \\
Ln RMSSD $(\mathrm{ms})$ & $4.42 \pm 0.43$ & -.50 & $0 / 49 / 51$, possibly negative & 0.21 \\
Ln LF $\left(\mathrm{ms}^{2}\right)$ & $7.05 \pm 0.77$ & .01 & $8 / 85 / 7$, unclear & 0.41 \\
Ln HF $\left(\mathrm{ms}^{2}\right)$ & $7.56 \pm 0.85$ & -.49 & $0 / 51 / 49$, possibly trivial & 0.35 \\
Ln LF/HF & $-0.51 \pm 0.77$ & .56 & $59 / 41 / 0$, possibly positive & 0.42 \\
Ln TP $\left(\mathrm{ms}^{2}\right)$ & $8.09 \pm 0.73$ & -.42 & $0 / 61 / 39$, possibly trivial & 0.34 \\
\hline
\end{tabular}

Note. $\quad r=$ Pearson's correlation coefficient; MBI = magnitude based inference for $r$; HRsup = heart rate in the supine position; $\mathrm{Ln}=$ natural logarithm; RMSSD $=$ square root of the mean of the squares of the successive differences; LF $=$ low-frequency power; $\mathrm{HF}=$ high-frequency power; $\mathrm{LF} / \mathrm{HF}=$ low-frequency/high-frequency ratio; $\mathrm{TP}=$ total power $(\mathrm{LF}+\mathrm{HF})$. 


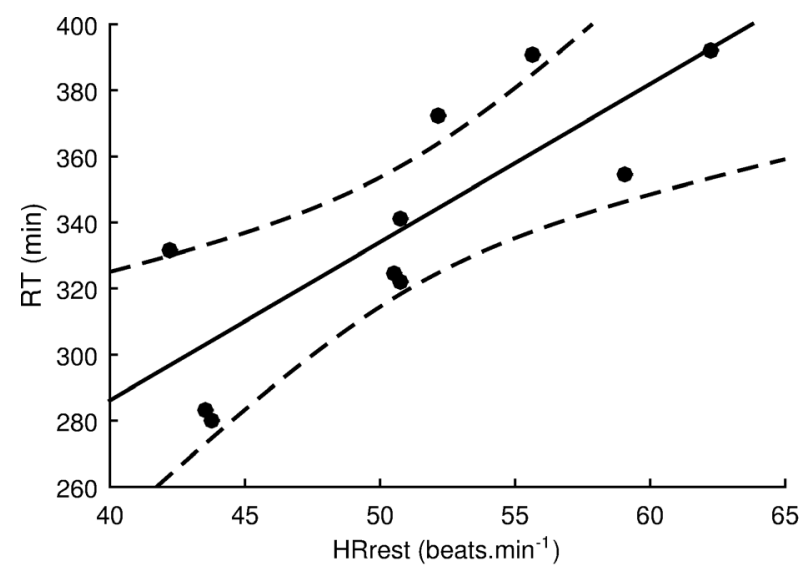

$r=.81, \mathrm{Cl}: .47$ to $.94, \mathrm{MBI}: 94 / 6 / 0$, likely positive

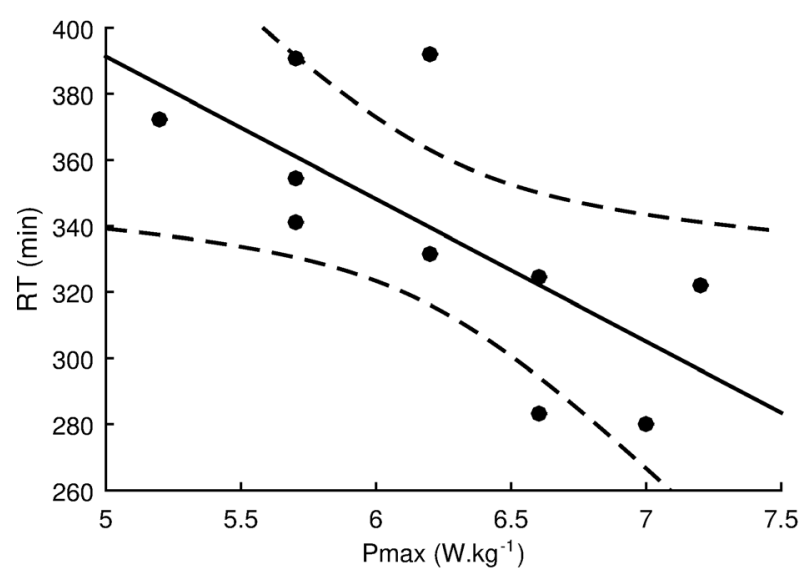

$r=-.70, \mathrm{Cl}:-.90$ to $-.25, \mathrm{MBI}: 0 / 20 / 80$, likely negative

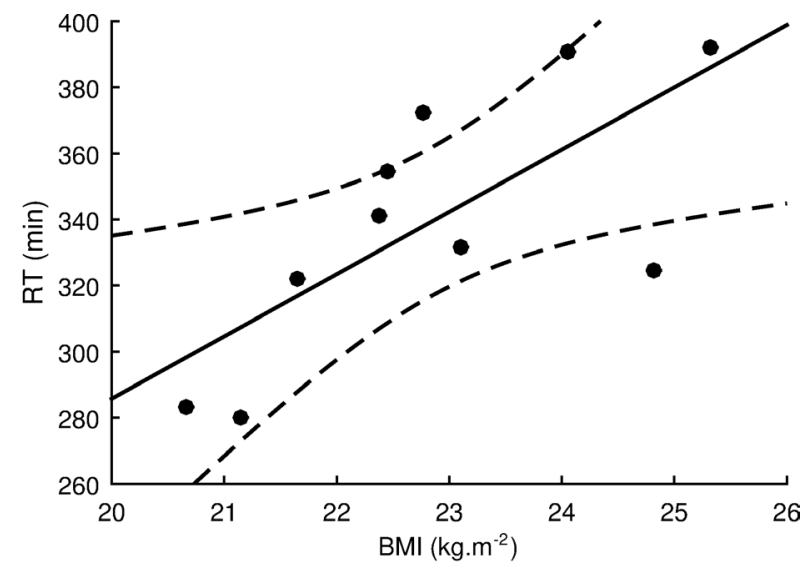

$r=.73, \mathrm{Cl}: .30$ to $.91, \mathrm{MBI}: 84 / 16 / 0$, likely positive

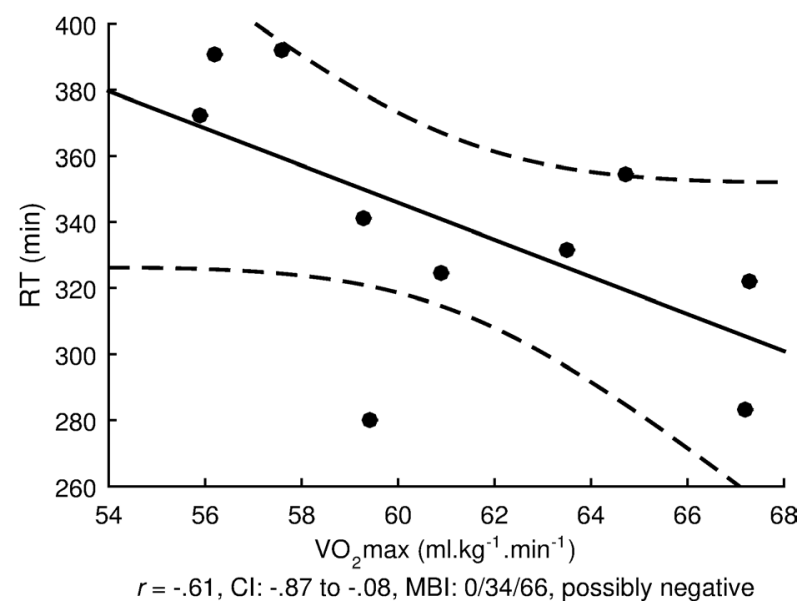

Figure 3. Correlation analysis between race time (RT) and selected variables. Dashed lines denote 0.90-confidence area. HRrest = morning heart rate in the supine position on the day of the race; $\mathrm{BMI}=$ body $\operatorname{mass}$ index; Pmax $=\max -$ imal power output; $\mathrm{VO}_{2} \max =$ maximal oxygen uptake; $r=$ Pearson's correlation coefficient; $\mathrm{CI}=0.90$-confidence interval for $r$; MBI = magnitude based inference that evaluates the chances that the true value of $r$ is substantially positive, trivial, or substantially negative.

an increase in sympathetic activity due to an excessive increase in training load in elite rowers was previously reported by Iellamo et al. (2002) who suggested that persistent sympathetic stimulation is as potential risk factor for the development of hypertension.

Relating to acute changes in autonomic regulation, Bernardi et al. (1997) reported that the sympathetic contribution to HR dominated in the first $30 \mathrm{~min}$ post a $46 \mathrm{~km}$ trail run performed at mild altitude, while $24 \mathrm{~h}$ post-race there was an increased sensitivity to vagal and reduced sensitivity to sympathetic baroreflex stimulation. In another study, elevated sympathetic activity (increased $\mathrm{LF} / \mathrm{HF}$ ) together with increased resting HR was found 6-8 h after a high-altitude marathon, whilst vagal activity restoration only occurred at $24 \mathrm{~h}$ post-race (Cornolo et al., 2005). However, contrary to the present findings, Hautala et al. (2001) showed that vagal activity, when expressed as HF in normalized units and Ln HF, returned to baseline after a $75 \mathrm{~km}$ cross-country skiing race, at $4.2 \pm 4.2 \mathrm{~h}$, and $8.2 \pm 5.4$ $\mathrm{h}$, respectively. In this context it has previously been shown that vagal recovery rate is related to aerobic fitness, with a higher $\mathrm{VO}_{2} \max$ associated with a faster recovery (Hautala et al., 2001; Seiler et al., 2007). Based on the higher aerobic capacity of our subjects $\left(\mathrm{VO}_{2} \max =60.5 \mathrm{ml} \cdot \mathrm{kg}^{-1} \cdot \mathrm{min}^{-1}\right)$ compared with the aerobic capacity $\left(\mathrm{VO}_{2} \max =51.0 \mathrm{ml} \cdot \mathrm{kg}^{-1} \cdot \mathrm{min}^{-1}\right)$ of the subjects in Hautala et al. (2001) study, we suggest that discrepancies between the two studies may be related to methodological issue rather than the aerobic fitness. In the present study, ANS was always assessed immediately after awakening pre- and post-race, compared with Hautala et al. (2001) who compared pre- and postrace HRV in their subjects by measuring vagal activity via recording $R R$ intervals in the evening and during sleep from 5 p.m. to 4 a.m. Importantly, vagal activity usually accumulates in healthy subjects during sleep (Ernst, 2014) and this may have influenced the vagal 

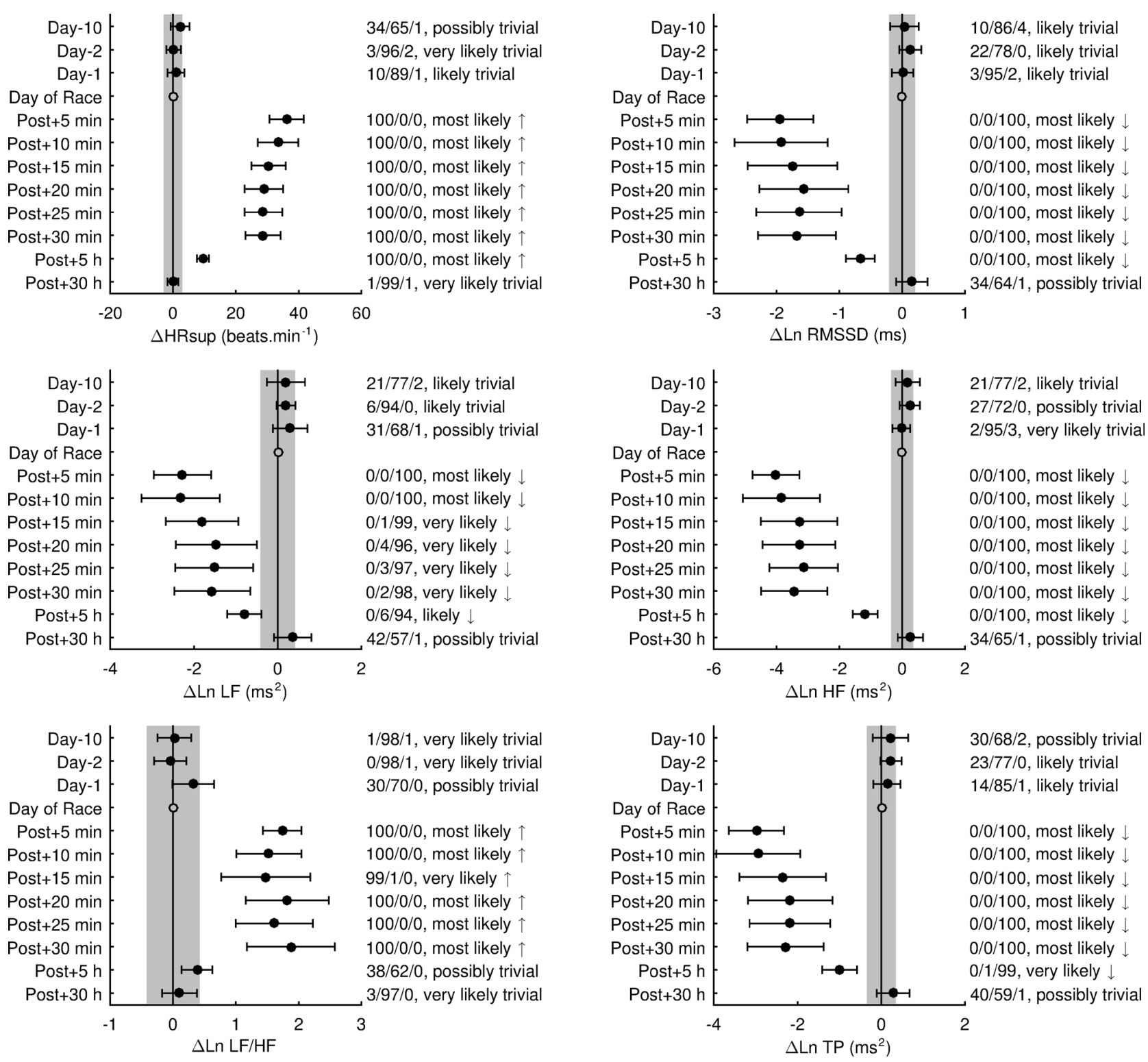

Figure 4. Changes in heart rate and heart rate variability. Values are presented as the mean and $90 \%$ confidence interval. Shaded area denotes trivial changes and was based on the smallest worthwhile change. $\Delta=$ difference between actual value and the morning value on the day of the race; HRsup = heart rate in the supine position; $\mathrm{Ln}=$ natural logarithm; RMSSD = square root of the mean of the squares of the successive differences; LF = low-frequency power; $\mathrm{HF}=$ high-frequency power; $\mathrm{LF} / \mathrm{HF}=$ low-frequency/high-frequency ratio; $\mathrm{TP}=$ total power $(\mathrm{LF}+\mathrm{HF}) ; \uparrow=$ substantial increase; $\downarrow=$ substantial decrease.

recovery rate and hence interpretation of the findings. Waking testing of ANS is currently the accepted protocol in field based HRV studies (Buchheit, 2014). Therefore, based on our findings we propose that heightened sympathetic contribution to cardiovascular regulation occurs for several hours after long-term extreme physical exertion such as SkyMarathon, while complete vagal activity restoration may be delayed by up to $30 \mathrm{~h}$ post. These findings are similar to those discussed in a recent review which reported that completed vagal recovery after vigorous endurance exercise may last at least $48 \mathrm{~h}$ (Stanley et al., 2013). From the point of view of ANS activity, athletes may be ready to perform another training after $30 \mathrm{~h}$ post SkyMarathon. However, it has been shown that cardiac vagal recovery is not able to reflect either metabolic or neuromuscular recovery (Stanley et al., 2013). It is clear that there are more factors involved than ANS alone that affect readiness to train. For example, Botek et al. (2008) showed that vagal activity was recovered up to $44 \mathrm{~h}$ post-run, whilst subjective fatigue (muscle stiffness, join stiffness, and painful walking) was significantly elevated 
over $58 \mathrm{~h}$ post-run. Therefore, it could be suggested that training recommendation should follow optimally a more than single body indicator.

It is well known that performance is influenced through the interaction of a number of variables. To the best of the authors knowledge, this is the first study to demonstrate a very large correlation $(r=.73)$ between BMI and RT in a SkyMarathon. Taking into account the undulating landscapes that are common in skyrunning races (Roi et al., 2015), a lower BMI at a given body fat percentage $(\sim 11 \%)$ seems to be advantageous for performance. Besides somatic characteristics, a very large correlation $(r=-.70)$ between Pmax (related to incremental test duration) and RT was detected. An interesting finding was that only a large correlation $(r=-.61)$ was found between $\mathrm{VO}_{2} \max$ and RT. Based on this finding, Pmax seems to be a better indicator of skyrunning performance than the $\mathrm{VO}_{2}$ max.

Buchheit et al. (2010) revealed a very large correlation $(r=.70)$ between vagally related index Ln RMSSD and running time in endurance running distances. We found only large correlation $(r=.50)$ between Ln RMSSD and RT but there was a very large correlation $(r=.81)$ between HRrest obtained on race day and RT. From this result it seems that runners with a lower resting HR on race day perform better. We suggest that sports bradycardia in nine of our runners (HR $<60$ beats $\cdot \mathrm{min}^{-1}$ ) may explain the discrepancies between correlations calculated from Ln RMSSD and resting HR. Specifically, low resting HR in elite athletes (below 50 beats $\cdot \mathrm{min}^{-1}$ ) has been linked with the HF power saturation phenomenon (Goldberger, Challapalli, Tung, Parker, \& Kadish, 2001; Kiviniemi, Hautala, Kinnunen, \& Tulppo, 2007; Plews et al., 2013). This saturation phenomenon has been shown to be responsible for masking the true vagal contribution to HR regulation. To avoid this methodological issue, it has repeatedly been recommended by some authors to use an orthoclinostatic challenge and not just a supine position when measuring HRV in well trained athletes (Botek, McKune, Krejci, Stejskal, \& Gaba, 2014; Kiviniemi et al., 2007).

The absence of a pre-race and post-race hydration status assessment can be considered a limitation of this study. Such data could provide better interpretation of HRV changes.

\section{Conclusion}

SkyMarathon reduced the cardiac vagal activity for at least $5 \mathrm{~h}$, while restoration occurred no later than $30 \mathrm{~h}$ after finishing the race. Based on Ln LF/HF variable, SkyMarathon increased relative cardiac sympathetic activity for at least $30 \mathrm{~min}$, while restoration occurred no later than $5 \mathrm{~h}$. From ANS viewpoint, the body is recovered after 30 hours of SkyMarathon and regular training can continue. This study found that resting $\mathrm{HR}$, BMI, Pmax, and $\mathrm{VO}_{2}$ max are good candidates for predictors of performance in SkyMarathon.

\section{Acknowledgment}

This study was supported by the Faculty of Physical Culture, Palacký University Olomouc, grant number IGA_FTK_2015_006.

\section{Conflict of interest}

There were no conflicts of interest.

\section{References}

Akselrod, S., Gordon, D., Ubel, F. A., Shannon, D. C., Berger, A. C., \& Cohen, R. J. (1981). Power spectrum analysis of heart rate fluctuation: A quantitative probe of beat-to-beat cardiovascular control. Science, 213, 220-222.

American College of Sports Medicine. (1986). Guidelines for exercise testing and prescription (3rd ed.). Philadelphia, PA: Lea \& Febiger.

Arai, Y., Saul, J. P., Albrecht, P., Hartley, L. H., Lilly, L. S., Cohen, R. J., \& Colucci, W. S. (1989). Modulation of cardiac autonomic activity during and immediately after exercise. American Journal of Physiology, 256, H132-H141.

Åstrand, P. O., Rodahl, K., Dahl, H. A., \& Strømme, S. B. (2003). Textbook of work physiology: Physiological bases of exercise. Champaign, IL: Human Kinetics.

Baron, B., Deruelle, F., Moullan, F., Dalleau, G., Verkindt, C., \& Noakes, T. D. (2009). The eccentric muscle loading influences the pacing strategies during repeated downhill sprint intervals. European Journal of Applied Physiology, 105, 749-757.

Batterham, A. M., \& Hopkins, W. G. (2006). Making meaningful inferences about magnitudes. International Journal of Sports Physiology and Performance, 1, 50-57.

Bernardi, L., Passino, C., Robergs, R., \& Appenzeller, O. (1997). Acute and persistent effects of a 46-kilometer wilderness trail run at altitude: Cardiovascular autonomic modulation and baroreflexes. Cardiovascular Research, 34, 273-280.

Botek, M., McKune, A. J., Krejci, J., Stejskal, P., \& Gaba, A. (2014). Change in performance in response to training load adjustment based on autonomic activity. International Journal of Sports Medicine, 35, 482-488.

Botek, M., Stejskal, P., Krejci, J., Jakubec, A., \& Gaba, A. (2010). Vagal threshold determination. Effect of age and gender. International Journal of Sports Medicine, 31, 768-772. 
Botek, M., Stejskal, P., \& Neuls, F. (2008). Monitoring of the autonomic nervous system activity during post-marathon recovery by spectral analysis of heart rate variability: A case study. Medicina Sportiva, 12, 31-35.

Botek, M., Stejskal, P., Šafář, M., \& Smékal, D. (2011). Autonomic nervous system activity assessment in recreational half marathon runners. Acta Universitatis Palackianae Olomucensis. Gymnica, 41, 7-14.

Buchheit, M. (2014). Monitoring training status with HR measures: Do all roads lead to Rome? Frontiers in Physiology, 5, 73.

Buchheit, M., Chivot, A., Parouty, J., Mercier, D., Al Haddad, H., Laursen, P. B., \& Ahmaidi, S. (2010). Monitoring endurance running performance using cardiac parasympathetic function. European Journal of Applied Physiology, 108, 1153-1567.

Buchheit, M., Papelier, Y., Laursen, P. B., \& Ahmaidi, S. (2007). Noninvasive assessment of cardiac parasympathetic function: Postexercise heart rate recovery or heart rate variability? American Journal of Physiology. Heart and Circulatory Physiology, 293, H8-H10.

Cornolo, J., Brugniaux, J. V., Macarlupu, J. L., Privat, C., León-Velarde, F., \& Richalet, J. P. (2005). Autonomic adaptations in Andean trained participants to a 4220-m altitude marathon. Medicine \& Science in Sports \& Exercise, 37, 2148-2153.

Dalla Vecchia, L., Traversi, E., Porta, A., Lucini, D., \& Pagani, M. (2014). On site assessment of cardiac function and neural regulation in amateur half marathon runners. Open Heart, 1, e000005.

Daniłowicz-Szymanowicz, L., Raczak, G., Pinna, G. D., Maestri, R., Ratkowski, W., Figura-Chmielewska, M., ... Ambrach-Dorniak, K. (2005). The effects of an extreme endurance exercise event on autonomic nervous system activity. Polski Merkuriusz Lekarski, 19, 28-31.

Ernst, G. (2014). Heart rate variability. London, United Kingdom: Springer-Verlag.

Flatt, A. A., Esco, M. R., \& Nakamura, F. Y. (2017). Individual heart rate variability responses to preseason training in high level female soccer players. Journal of Strength and Conditioning Research, 31, 531-538.

Fridén, J., \& Lieber, R. L. (2001). Eccentric exercise-induced injuries to contractile and cytoskeletal muscle fibre components. Acta Physiologica Scandinavica, 171, 321-326.

Goldberger, J. J., Challapalli, S., Tung, R., Parker, M. A., \& Kadish, A. H. (2001). Relationship of heart rate variability to parasympathetic effect. Circulation, 103, 1977-1983.

Hautala, A. J., Mäkikallio, T. H., Kiviniemi, A., Laukkanen, R. T., Nissilä, S., Huikuri, H. V., \& Tulppo, M. P. (2003). Cardiovascular autonomic function correlates with the response to aerobic training in healthy sedentary subjects. American Journal of Physiology. Heart and Circulatory Physiology, 285, H1747-H1752.

Hautala, A., Tulppo, M. P., Mäkikallio, T. H., Laukkanen, R., Nissilä, S., \& Huikuri, H. V. (2001). Changes in cardiac autonomic regulation after prolonged maximal exercise. Clinical Physiology, 21, 238-245.

Hopkins, W. G. (2007). A spreadsheet for deriving a confidence interval, mechanistic inference and clinical inference from a p value. Sportscience, 11, 16-20.
Hopkins, W. G., Marshall, S. W., Batterham, A. M., \& Hanin, J. (2009). Progressive statistics for studies in sports medicine and exercise science. Medicine \& Science in Sports \& Exercise, 41, 3-13.

Iellamo, F., Legramante, J. M., Pigozzi, F., Spataro, A., Norbiato, G., Lucini, D., \& Pagani, M. (2002). Conversion from vagal to sympathetic predominance with strenuous training in high-performance world class athletes. Circulation, 105, 2719-2724.

International Skyrunning Federation. (2017). Skyrunning rules. La Massana, Andorra: International Skyrunning Federation.

Kaikkonen, P., Hynynen, E., Mann, T., Rusko, H., \& Nummela, A. (2010). Can HRV be used to evaluate training load in constant load exercises? European Journal of Applied Physiology, 108, 435-442.

Kiviniemi, A. M., Hautala, A. J., Kinnunen, H., \& Tulppo, M. P. (2007). Endurance training guided individually by daily heart rate variability measurements. European Journal of Applied Physiology, 101, 743-751.

Millet, G. P., Libicz, S., Borrani, F., Fattori, P., Bignet, F., \& Candau, R. (2003). Effects of increased intensity of intermittent training in runners with differing $\mathrm{VO}_{2}$ kinetics. European Journal of Applied Physiology, 90, 50-57.

Mourot, L., Fabre, N., Andersson, E., Willis, S., Buchheit, M., \& Holmberg, H. C. (2015). Cross-country skiing and postexercise heart-rate recovery. International Journal of Sports Physiology and Performance, 10, 11-16.

Nakamura, F. Y., Flatt, A. A., Pereira, L. A., RamirezCampillo, R., Loturco, I., \& Esco, M. R. (2015). Ultrashort-term heart rate variability is sensitive to training effects in team sports players. Journal of Sports Science and Medicine, 14, 602-605.

Noakes, T. D. (2000). Physiological models to understand exercise fatigue and the adaptations that predict or enhance athletic performance. Scandinavian Journal of Medicine and Science in Sports, 10, 123-145.

Penttilä, J., Helminen, A., Jartti, T., Kuusela, T., Huikuri, H. V., Tulppo, M. P., ... Scheinin, H. (2001). Time domain, geometrical and frequency domain analysis of cardiac vagal outflow: Effects of various respiratory patterns. Clinical Physiology, 21, 365-376.

Perini, R., Orizio, C., Baselli, G., Cerutti, S., \& Veicsteinas, A. (1990). The influence of exercise intensity on the power spectrum of heart rate variability. European Journal of Applied Physiology and Occupational Physiology, 61, 143-148.

Plews, D. J., Laursen, P. B., Stanley, J., Kilding, A. E., \& Buchheit, M. (2013). Training adaptation and heart rate variability in elite endurance athletes: Opening the door to effective monitoring. Sports Medicine, 43, 773-781.

Pomeranz, B., Macaulay, R. J., Caudill, M. A., Kutz, I., Adam, D., Gordon, D., .... Benson, H. (1985). Assessment of autonomic function in humans by heart rate spectral analysis. American Journal of Physiology. Heart and Circulatory Physiology, 248, H151-H153.

Roi, G. S., Monticone, M., Salvoni, M., Sassi, R., \& Alberti, G. (2015). Self-reported knee symptoms assessed by KOOS questionnaire in downhill runners (skyrunners). PLoS ONE, 10, e0126382. 
Seiler, S., Haugen, O., \& Kuffel, E. (2007). Autonomic recovery after exercise in trained athletes: Intensity and duration effects. Medicine \& Science in Sports \& Exercise, 39, 1366-1373.

Shephard, R. J., \& Åstrand, P. O. (1992). Endurance in sport. Oxford, United Kingdom: Blackwell Science.

Stanley, J., Peake, J. M., \& Buchheit M. (2013). Cardiac parasympathetic reactivation following exercise: Implications for training prescription. Sports Medicine, 43, 1259-1277.

Stejskal, P., Rechbergová, J., Salinger, J., Šlachta, R., Elfmark, M., Kalina, M., ... Rehová, I. (2001). Power spectrum of heart rate variability in exercising humans: The effect of exercise intensity. Sports Medicine, Training and Rehabilitation, 10, 39-57.
Task Force of the European Society of Cardiology and the North American Society of Pacing and Electrophysiology. (1996). Heart rate variability: Standards of measurement, physiological interpretation and clinical use. Circulation, 93, 1043-1065.

Vernillo, G., Savoldelli, A., Zignoli, A., Trabucchi, P., Pellegrini, B., Millet, G. P., \& Schena, F. (2014). Influence of the world's most challenging mountain ultra-marathon on energy cost and running mechanics. European Journal of Applied Physiology, 114, 929-939.

Warren, J. H., Jaffe, R. S., Wraa, C. E., \& Stebbins, C. L. (1997). Effect of autonomic blockade on power spectrum of heart rate variability during exercise. American Journal of Physiology. Regulatory, Integrative and Comparative Physiology, 273, R495-R502. 